\title{
New Science with Atom Probe Tomography Enabled via an Environmental Transfer Hub
}

\author{
Mark G. Wirth ${ }^{1 *}$ and Daniel E. Perea ${ }^{1 *}$ \\ 1. Environmental Molecular Sciences Laboratory, Pacific Northwest National Laboratory, Richland, \\ WA, USA \\ * Corresponding authors: mark.wirth@pnnl.gov, daniel.perea@pnnl.gov
}

Atom probe tomography (APT) is a technique that allows 3-dimensional (3D) imaging and chemical composition measurements at the atomic scale. Specimens for APT analysis are materials sharpened to a very sharp tip. This tip is biased with a large DC voltage and pulsed with either a laser or high voltage. Atoms are evaporated from the surface and are detected using a position sensitive time of flight (TOF) mass spectrometer. The information from the mass spectrometer can be reconstructed to create a 3D image of the tip [1].

APT often requires the use of dual beam scanning electron microscope/focused ion beam (FIB/SEM) for sample preparation. Transferring samples from FIB/SEM to APT for analysis presents a challenge for samples that are sensitive to ambient air and room temperature, requiring such environmentally-sensitive materials to either be prepared in situ or transported within the controlled environment of a specimen shuttle device.

A unique Environmental Transfer Hub (ETH) (Fig. 1a) has been designed and fabricated at PNNL and directly interfaced with a Local Electrode Atom Probe (CAMECA LEAP 400X HR) [2]. The ETH consists of a central Ultra High Vacuum (UHV) chamber which connects directly to the buffer chamber on the LEAP. The ETH has a load lock assembly to connect a specimen shuttle device (suitcase), allowing the transfer of samples from FIB/SEM to the LEAP under environmentally-controlled temperature and pressure conditions; cryogenic to room temperature and high vacuum to inert atmosphere respectively (Fig. 1b) [2].

The suitcase is a modified Quorum PT 2010T shuttle device (Fig. 1c) with a custom sample puck manipulator (puckulator) fabricated from polyether ether ketone plastic designed to reduce conductive thermal transfer. A custom cold stage adapter was fitted to the Quorum PT 2010T cryo-FIB cold stage (Fig. 1d) to accept the unique LEAP specimen pucks. The stage adapter is made from copper and has an integrated thermocouple and heater that allows temperature control between 81-370 K [3].

The design of the ETH is such that it allows for the interfacing of other ancillary tools such as a high temperature variable pressure reactor. The combination of the ETH and the reactor are providing a unique means to handle environmentally-sensitive materials, thus allowing the APT technique to access new areas of science (Fig. 1e). For example: 1) the reactor provides a capability to perform temperature and pressure-controlled oxidation and reduction surface reactions on pristine specimen surfaces for analysis with APT via in situ UHV specimen transfer; 2) ability to prepare and transfer specimens under cryogenic conditions provides a means to analyze ionic and macromolecular gradients in organic and inorganic hydrated systems, as well as 3) prepare specimens from pristine molecules that can be analyzed to provide fundamental insight into field evaporation during APT analysis. 


\section{References:}

[1] Devaraj et al, Int Mat Rev, 63 (2017) p. 68-101

[1] D.E. Perea et al, Adv Struct Chem Imag, (2017) p. 3-12.

[2] D.K. Schreiber et al, Ultramicroscopy, 194 (2018) p. 89-99.

[3] This work was performed using EMSL, a national scientific user facility sponsored by the Department of Energy's Office of Biological and Environmental Research and located at PNNL.
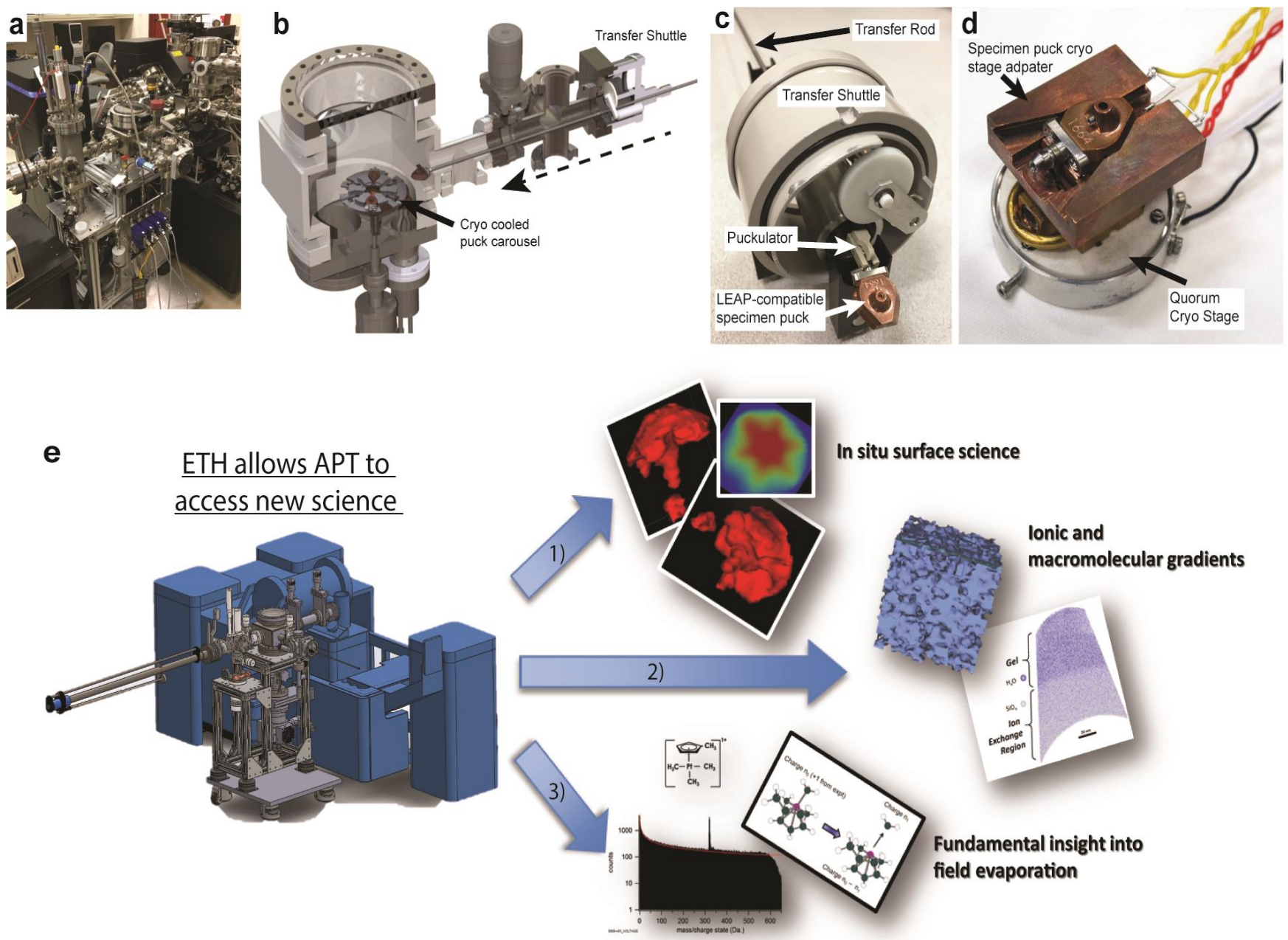

Figure 1. The ETH design and configuration. a Photograph of the ETH system connected to the LEAP at Pacific Northwest National Laboratory. b CAD-rendered image of the ETH main vacuum hub with the specimen shuttle docked. The shuttle transfer rod with LEAP specimen puck is extended toward a cryo-cooled puck carousel. c Photograph of the transfer shuttle also known as the suitcase. d Photograph of the customized FIB/SEM cold stage with LEAP specimen puck inserted. e Image illustrating some of the new branches of science that can be accessed by APT in conjunction with the ETH [2]. 